\title{
WEYL LAWS ON OPEN MANIFOLDS
}

\author{
SERGIU MOROIANU
}

\begin{abstract}
Under suitable invertibility hypothesis, the spectrum of the Dirac operator on certain open spin Riemannian manifolds is discrete, and obeys a growth law depending qualitatively on the (in)finiteness of the volume.
\end{abstract}

\section{INTRODUCTION}

Let $M$ be a closed Riemannian manifold, $\mathcal{E} \rightarrow M$ a Hermitian vector bundle and $D: \mathcal{C}^{\infty}(M, \mathcal{E}) \rightarrow \mathcal{C}^{\infty}(M, \mathcal{E})$ an elliptic, symmetric, positive differential operator of order $k>0$. Then the spectrum of $D$ is discrete and the eigenvalues accumulate towards infinity obeying the Weyl law:

$$
\lim _{\lambda \rightarrow \infty} \frac{N(\lambda)}{\lambda^{\operatorname{dim}(M) / k}}=C
$$

where $N(\lambda):=\#\{s \in \operatorname{Spec}(D) ;|s|<\lambda\}$ is the counting function and $C$ is an explicit constant depending on $\operatorname{dim}(M)$ and on the principal symbol of $D$. This fact is classically proved using the heat trace expansion [25] when $D$ is a Laplacian. Note that one can obtain better estimates of the remainder in (1), see [13].

In this paper we derive similar laws for Dirac operators on certain open spin Riemannian manifolds.

If the manifold $M$ is not compact, not much can be said in general about the spectrum of $D$. However, if $M$ is complete then the Laplacian on forms and the Dirac operator are essentially self-adjoint, and their essential spectra may be non-empty. Several results are also known for compact, incomplete manifolds with boundary. The original result of Weyl states that (1) holds for the Dirichlet Laplacian on a compact domain with smooth boundary in $\mathbb{R}^{n}$. Let now $\bar{X}$ be a compact manifold with boundary $M$, and $X$ its interior. Then (1) holds [3] for the Dirac

Date: October 31, 2018.

2000 Mathematics Subject Classification. 58G50.

The author has been partially supported by the Research and Training Network HPRN-CT-1999-00118 "Geometric Analysis" funded by the European Commission. 
operator on $X$ endowed with a product-type metric near $M$ and a suitable non-local boundary condition. The results of [3] follow from an explicit computation on the cylinder, which breaks down for warped product metrics. A significant progress in this context was brought by Melrose [22], who computed the index of the Atiyah-Patodi-Singer non-local boundary value problem as an $L^{2}$ index, using his b-calculus on the complete manifold obtained from $\bar{X}$ by gluing infinite cylinders. Unlike the APS operator, the associated b-operator has non-empty essential spectrum.

The present work is motivated by a result of Bär [4], who showed that the essential spectrum of the Dirac operator on complete spin hyperbolic manifolds of finite volume is either empty or the whole real line. Thus, let $\bar{X}$ be a smooth $n$-dimensional compact manifold with closed boundary $M$, and $x$ a boundary-defining function. This means

- $x \in \mathcal{C}^{\infty}(\bar{X},[0, \infty))$;

- $M=\{x=0\}$

- $d x$ never vanishes at $x=0$.

Let $g_{0}$ be a cusp metric on $\bar{X}$, i.e., a (complete) Riemannian metric on $X:=\bar{X} \backslash M$ which in local coordinates near the boundary takes the form

$$
g_{0}=a_{00}(x, y) \frac{d x^{2}}{x^{4}}+\sum_{j=1}^{n-1} a_{0 j}(x, y) \frac{d x}{x^{2}} d y_{j}+\sum_{i, j=1}^{n-1} a_{i j}(x, y) d y_{i} d y_{j}
$$

such that the matrix $A=\left(a_{\alpha \beta}\right)$ is smooth and non-degenerate down to $x=0$. Let $h:=\sum_{i, j=1}^{n-1} a_{i j}(0, y) d y_{i} d y_{j}$ denote the induced Riemannian metric on $M$. For simplicity, in this introduction we present our results under the additional assumption

$$
a_{00 \mid M} \equiv 1, \quad a_{0 j \mid M} \equiv 0 .
$$

Following Melrose [22], we call $g_{0}$ satisfying (3) an exact cusp metric. We are interested in the Riemannian metrics

$$
g_{p}:=x^{2 p} g_{0}
$$

for $p \in \mathbb{R}$. Important particular cases are obtained when $g_{0}$ is a cylindrical metric on $(0, \epsilon) \times M \subset X, g_{0}=x^{-4} d x^{2}+h$. By the change of variable $y^{q}=x, q>0$ (which changes the smooth structure of $\bar{X}$ but not that of $X$ ) and for varying $p \in \mathbb{R}$ we can get any metric of the type

$$
x^{a} d x^{2}+x^{b} h
$$


with $a, b \in \mathbb{R}$ provided $b-a>2$, in particular $g_{p}$ cannot be conformally conical. Such metrics include all metric horns [18], and complete hyperbolic manifolds of finite volume. The above change of variable is rather artificial since it destroys smoothness of coefficients in the general case (2).

We first study the self-adjointness of the Dirac operator of the metric $g_{p}$ acting on spinors.

Theorem 1. Let $X$ have a spin structure such that the Dirac operator $D^{h}$ for the induced spin structure on $(M, h)$ is invertible. Assume that $g_{0}$ satisfies (3). Then the Dirac operator $D_{p}$ with domain $\mathcal{C}_{c}^{\infty}(X, \Sigma)$ is essentially self-adjoint in $L^{2}\left(X, \Sigma, g_{p}\right)$.

This result is standard for $p \leq 1$ even without the invertibility hypothesis, since then $g_{p}$ is complete. For $p>1$ and $g_{0}=x^{-4} d x^{2}+h$ we recover a result from [18] through the change of variable $y=x^{p-1}$. For us the most interesting phenomena will occur for $0<p \leq 1 / n$.

It is important to understand how restrictive the invertibility hypothesis really is. On one hand, it is conjectured that any spin manifold $M$ admits metrics with harmonic spinors, provided $\operatorname{dim}(M) \geq 3$. On the other hand, it is also conjectured that (for $M$ connected) for generic metrics the dimension of the space of harmonic spinors is the minimal dimension prescribed by index theory. Parts of this conjecture were proved by Maier [20] and Bär and Dahl [5]. In our case, by the cobordism invariance of the index, the minimal dimension is 0 . Thus, for $M$ connected, the invertibility hypothesis is automatically satisfied for generic metrics if $\operatorname{dim}(M) \leq 4$, or if $\pi_{1}(M)$ vanishes or is cyclic of odd order. An indirect way of ensuring invertibility of $D^{h}$ is (following Lichnerowicz) asking that $\mathrm{scal}_{h} \geq 0$, with strict inequality at least at one point.

Incidentally, under the hypothesis of Theorem $1, D_{p}^{+}$is in fact Fredholm, and for $\operatorname{dim} X$ even,

$$
\operatorname{index}\left(D_{p}^{+}\right)=\int_{\bar{X}} \hat{A}\left(g_{p}\right)-\eta\left(D^{h}\right) / 2 .
$$

The $\hat{A}$ form is a conformal invariant, hence, like $\operatorname{index}\left(D_{p}^{+}\right)$, it is independent of $p$. We will not pursue this fact further.

Next, we look for conditions on $p$ to ensure the vanishing of the essential spectrum of $D_{p}$. 
Theorem 2. Under the hypothesis of Theorem 1, the operator $D_{p}$ has pure point spectrum if and only if $p>0$.

This greatly generalizes the above cited result of Bär [4].

Our main result describes the asymptotics of the counting function for the eigenvalues of $D_{p}$.

Theorem 3. Let $\bar{X}$ be a compact $n$-dimensional spin manifold with boundary $M, g_{p}$ the metric on $X$ given by (4) and $D_{p}$ the associated Dirac operator on spinors. Assume that (3) holds and that the spin structure on $\bar{X}$ induces an invertible Dirac operator on $(M, h)$. For $p>0$ let $N_{p}(\lambda)$ denote the counting function for the eigenvalues of $D_{p}$ (well-defined by Theorem 2). Then

- For $1 / n<p<\infty$,

$$
N_{p}(\lambda) \approx \lambda^{n} \frac{\operatorname{Vol}\left(X, g_{p}\right) \operatorname{Vol}\left(S^{n-1}\right) 2^{[n / 2]}}{n(2 \pi)^{n}} .
$$

- For $p=1 / n$,

$$
N_{p}(\lambda) \approx \lambda^{n} \log \lambda \frac{\operatorname{Vol}(M, h) \operatorname{Vol}\left(S^{n-1}\right) 2^{[n / 2]}}{(2 \pi)^{n}} .
$$

- For $0<p<1 / n$,

$$
N_{p}(\lambda) \approx \lambda^{1 / p} \frac{\Gamma\left(\frac{1-p}{2 p}\right) \zeta\left(D^{h}, \frac{1}{p}-1\right) \epsilon_{n}}{2 \sqrt{\pi} \Gamma\left(\frac{1}{2 p}\right)},
$$

where $\epsilon_{n}$ equals 1 if $n$ is odd, 2 if $n$ is even.

Here $\Gamma$ is the Gamma function and $\zeta\left(D^{h}, z\right):=\operatorname{Tr}\left(\left(D^{h}\right)^{2}\right)^{-z / 2}$ is the zeta function of the absolute value of $D^{h}$, which is holomorphic for $\Re(z)>n-1=\operatorname{dim}(M)$.

It is interesting to note that $\left.\operatorname{Vol}\left(X, g_{p}\right)\right)$ is finite exactly when it arises as coefficient in the asymptotic law. In that case the formula bears no difference from the case of closed manifolds. The critical conformal factor $p=1 / n$ marks the start of a remarkable change in the growth rate. As $p$ approaches 0 , the metric becomes close to the asymptotically cylindrical metric, for which the essential spectrum does not vanish. This is reflected by the growth rate becoming infinite. Remarkably, some sort of spectral asymptotics for the Laplacian survive for $g_{0}$, as shown by Christiansen and Zworski [8].

Weyl asymptotics may be deduced by two related approaches - heat trace expansions, via the beautiful and elementary result of Karamata, 
and from meromorphic extensions of zeta functions via the Ikehara theorem. Complex powers are weaker but more conceptual than the heat kernel, in particular they can be constructed in wide generality [1] following the method of Guillemin [11] and Bucicovschi [7]. Thus our strategy of proof will be to construct the complex powers of the square of our Dirac operators inside a calculus of pseudodifferential operators, and examine the leading pole of the associated zeta function. Rather than constructing a different calculus for each $p$, which would be highly problematic, we rely on Melrose's cusp calculus, corresponding to $p=0$ (note that geometrically it does not correspond to cuspidal but to cylindrical ends). For each $p$ we find an isometry between $L^{2}\left(\Sigma_{p}\right)$ and $L^{2}\left(\Sigma_{0}\right)$ under which $D_{p}$ transforms to a cusp operator of order $(1, p)$. The zeta function of this operator is a meromorphic function, with first pole at $\max \{n, 1 / p\}$, double if $n=1 / p$ and simple otherwise. We use then the Ikehara theorem [14] and its generalization by Delange [9] to deduce Theorem 3. The invertibility of $D^{h}$ means that the operator $D_{p}$ is fully elliptic, hence determines the domain of its closure and yields Theorem 1. As for Theorem 2, the condition $p>0$ is equivalent to the parametrix of $D_{p}$ inside the cusp calculus being compact.

It might be possible to retrieve our results by exhibiting small-time asymptotics of the heat kernel of $D_{p}^{2}$. Paul Loya (see e.g., [19]) has constructed such heat kernels and studied their asymptotics in a variety of non-standard situations. Such asymptotics would be stronger than the meromorphy of the zeta function; in exchange, they would yield immediately Theorem 3 via Karamata's theorem.

Let us mention a third possible approach, i.e., the asymptotics of the wave trace. Even the first step of identifying this trace with a tempered distribution depends mildly on some a priori knowledge of Weyl asymptotics. Nevertheless, this approach would likely give also an estimate of the error term in Theorem 3.

We finally mention without proof that our results seem to extend to manifolds with corners of codimension $d \geq 2$. For various conformal factors, one gets as possible growth rates $\lambda^{n} \log ^{k} \lambda, k=0, \ldots, d$, and also $\lambda^{1 / p} \log ^{k} \lambda, 0<p<1 / n, k=0, \ldots, d-1$.

Acknowledgments. I wish to thank Andrei Moroianu for several useful discussions. 


\section{The CUSP STRUCTURE}

Consider a general cusp metric given by (2) without the condition (3), and the closely related metrics on $X$ obtained by the conformal transformation of weight $x^{p}, p \in \mathbb{R}$. We call the metric $g_{p}=x^{2 p} g_{0}$ a $p$-metric. Note that the volume of $g_{p}$ is finite if and only if $p>1 / n$.

Example 4. A complete hyperbolic manifold $X$ of finite volume is isometric (outside a compact set) to a warped product cylinder

$$
\left((-\infty, 0] \times M, d t^{2}+e^{-2 t} h\right)
$$

where $M$ may be disconnected. Moreover, $h$ is flat and independent of $t$. By the change of variables $x=e^{-t}$ near $-\infty$, the hyperbolic metric transforms to $x^{2} h+\frac{d x^{2}}{x^{2}}$ near $x=0$. Thus a hyperbolic metric of finite volume is a 1-metric on the compactification $\bar{X}:=X \cup\{-\infty\} \times M$.

Let

$$
{ }^{c} \mathcal{V}(\bar{X}):=\left\{V \in \mathcal{C}^{\infty}(\bar{X}, T \bar{X}) ; d x(V) \in x^{2} \mathcal{C}^{\infty}(\bar{X}, T \bar{X})\right\} \subset \mathcal{V}(\bar{X})
$$

be the Lie sub-algebra of cusp vector fields on $\bar{X}$. In local coordinates $\left(x, y_{j}\right)$ near $x=0$, a vector field in ${ }^{c} \mathcal{V}(\bar{X})$ can be uniquely written as a linear combination

$$
a(x, y) x^{2} \partial_{x}+\sum_{j=1}^{n-1} b_{j}(x, y) \partial_{y_{j}}
$$

with smooth coefficients $a, b_{j}$. Thus ${ }^{c} \mathcal{V}(\bar{X})$ is a locally free $\mathcal{C}^{\infty}(\bar{X})$ module. By the Serre-Swan theorem there exists a vector bundle $T \bar{X} \rightarrow \bar{X}$ such that ${ }^{c} \mathcal{V}(\bar{X})=\mathcal{C}^{\infty}(\bar{X}, T \bar{X})$. Moreover, the inclusion ${ }^{c} \mathcal{V}(\bar{X}) \hookrightarrow \mathcal{C}^{\infty}(\bar{X}, T \bar{X})$ gives rise to a bundle map

$$
c:{ }^{\natural} T \bar{X} \rightarrow T \bar{X}
$$

which is an isomorphism outside $x=0$. A curious feature of the cusp tangent bundle is that it has a canonical normal subbundle to the boundary, rather than conormal as in the standard case. Namely, the vector field $x^{2} \partial_{x}$ is well-defined (and non-vanishing) regardless of changes in the other local coordinates $y_{1}, \ldots, y_{n-1}$.

Lemma 5. A metric $g_{0}$ on $X$ extends to a Riemannian metric on the bundle $T \bar{X} \rightarrow \bar{X}$ if and only if it has the form (2).

Proof. Clearly, cusp metrics have been defined so that this lemma holds. 
In particular, $g_{0}$ takes the same form for different local coordinates $y_{j}$, which might not have been a priori clear. Notice that near $M$ we can write

$$
g_{0}=a_{00} \frac{d x^{2}}{x^{4}}+\frac{d x}{x} \otimes \theta^{X}+\theta^{X} \otimes \frac{d x}{x}+h^{X}
$$

where $\theta^{X}$ and $h^{X}$ are a smooth 1-form, respectively a symmetric 2tensor which restricts to be non-degenerate on the level sets $\{x=$ constant $\}$. There is an ambiguity in the definition of $\theta^{X}, h^{X}$ which can be removed by choosing a smooth extension $Z_{0}$ of the canonical vector field $x^{2} \partial_{x}$ to $\bar{X}$ such that $d x\left(Z_{0}\right)=x^{2}$, and asking that $\theta^{X}, h^{X}$ vanish on $Z_{0}$. This vector field corresponds to a product decomposition of a neighborhood of $M$.

Let now $x^{\prime}=a x+B x^{2}$, with $a \in \mathcal{C}^{\infty}\left(M, \mathbb{R}_{+}^{*}\right), B \in \mathcal{C}^{\infty}(\bar{X}, \mathbb{R})$, be the Taylor limited development of another boundary-defining function $x^{\prime}$. Then $x^{\prime}$ and $x$ define the same Lie algebra of cusp vector fields if and only if $a$ is a constant. The set of such functions is called a cusp structure, which is henceforth fixed. However, it proves useful not to fix the function $x$ inconsiderately inside the cusp structure since by Lemma 5 , the metric $g_{0}$ is also a cusp metric with respect to $x^{\prime}$. The canonical vector fields $x^{2} \partial_{x}$ and $x^{\prime 2} \partial_{x^{\prime}}$ are related by $x^{2} \partial_{x}=a^{-1} x^{\prime 2} \partial_{x^{\prime}}$, thus the cusp normal bundle is canonically trivialized up to a constant.

Let $\mathcal{I}$ be the Lie ideal $x \cdot{ }^{c} \mathcal{V}(\bar{X})$ inside ${ }^{c} \mathcal{V}(\bar{X})$. We also denote by $\mathcal{I}$ the space $x \mathcal{C}^{\infty}\left(\bar{X}, T^{*} \bar{X}\right)$, and more generally any ideal of the form $x \mathcal{C}^{\infty}(\bar{X}, E)$ where $E$ is a smooth vector bundle over $\bar{X}$.

Lemma 6. Let $g_{0}$ be a cusp metric written in the form (5). Let $h:=$ $h_{\mid M}^{X}, \theta:=\theta_{\mid M}^{X}$ and $q:=\left.a_{00}\right|_{M} \in \mathcal{C}^{\infty}(M)$. Define $a 1$-form $\alpha$ and $a$ metric $g^{M}$ on $M$ by

$$
\alpha:=\frac{\theta}{q}, \quad g^{M}:=\frac{h}{q}-\alpha \otimes \alpha .
$$

Then $q, g^{M}$ and the residue class $\alpha+d \Lambda^{0}(M)$ are independent, up to a multiplicative constant, of the boundary-defining function $x$ inside the fixed cusp structure.

Proof. There is an ambiguity in the choice of $\theta^{X}$ and $h^{X}$ but only up to $\mathcal{I}^{2}$, so $\theta$ and $h$ are well-defined. Clearly, $0<\left\|x^{2}-\theta^{\#}\right\|_{g_{0}}^{2}=q-\|\theta\|_{h}^{2}$ so $g^{M}$ is non-degenerate. We write

$$
g_{0}=a_{00}\left(\left(\frac{d x}{x^{2}}+\frac{\theta^{X}}{a_{00}}\right)^{2}+\frac{h^{X}}{a_{00}}-\frac{\theta^{X}}{a_{00}} \otimes \frac{\theta^{X}}{a_{00}}\right) .
$$


Let $x^{\prime}$ be another boundary-defining function in the cusp structure of $x$, i.e., $x=a x^{\prime}+B x^{\prime 2}$ with $a>0$ constant, $B \in \mathcal{C}^{\infty}(\bar{X})$. Then

$$
\frac{d x}{x^{2}}=\frac{1}{a} \frac{d x^{\prime}}{x^{\prime 2}}+\frac{d B}{a^{2}}+\mathcal{I}
$$

so $q^{\prime}=\frac{q}{a^{2}}, \alpha^{\prime}=a \alpha+\frac{d b}{a}$, where $b=B_{\mid M}$, and the lemma follows.

Melrose [22] calls cusp metrics with property (3) exact. In light of the above lemma, a cusp metric with $a_{00 \mid M} \equiv 1$ and $\theta$ exact can be put in the form (3). By the Hodge decomposition, we can always modify $x$ so that $\alpha$ is coexact with respect to $g^{M}$. It makes sense therefore to distinguish closed cusp metrics as being those with $\theta / q$ closed; in that case, a change of boundary-defining function can make $\theta / q$ harmonic with respect to $g^{M}$.

The invariant $\alpha+d \Lambda^{0}(M)$ has no equivalent in the case of finite-length cylinders, see [6].

\section{3. $p$ - VERSUS 0-DIRAC OPERATORS}

Assume that $\left(X, g_{0}\right)$ has a spin structure $\pi: P_{\text {Spin }}(X) \rightarrow P_{S O}\left(X, g_{0}\right)$. Let $T_{p}:\left(T X, g_{p}\right) \rightarrow\left(T X, g_{0}\right)$ be the isometry defined by $V \mapsto x^{p} V$. It induces a $S O(n)$-isomorphism

$$
T: P_{S O}\left(X, g_{p}\right) \rightarrow P_{S O}\left(X, g_{0}\right)
$$

between the orthonormal frame bundles of $\left(X, g_{p}\right)$ and $\left(X, g_{0}\right)$. We define a spin structure on $\left(X, g_{p}\right)$ by $T^{-1} \pi: P_{\text {Spin }}(X) \rightarrow P_{S O}\left(X, g_{p}\right)$. Thus the cusp- and $p$-spinors have the same underlying vector bundle $\Sigma$, with the same metrics but different Clifford module structures. The Clifford action $c^{g_{p}}$ with respect to $g_{p}$ obeys the rule

$$
c^{g_{p}}(V) \phi=c^{g_{0}}(T(V)) \phi .
$$

Proposition 7. The unbounded operator $D_{p}$ in $L^{2}\left(X, \Sigma, g_{p}\right)$ with domain $\mathcal{C}_{c}^{\infty}(X, \Sigma)$ is isometric to

$$
A_{p}=x^{-p}\left(D_{0}-\frac{p}{2} x c^{0}\left(\frac{d x}{x^{2}}\right)\right)
$$

acting in $L^{2}\left(X, \Sigma, g_{0}\right)$ with domain $\mathcal{C}_{c}^{\infty}(X, \Sigma)$.

Proof. Note that $d x / x^{2}$ is a smooth section in ${ }^{T} T^{*} \bar{X}$ over $\bar{X}$, thus $c^{0}\left(d x / x^{2}\right)$ is actually non-singular down to $x=0$. 
For every conformal transformation $\tilde{g}=f^{2} g$, the Dirac operators $D_{\tilde{g}}$ and $D_{g}$ are related by [12, Prop. 1.3]:

$$
D_{\tilde{g}}=f^{-\frac{n+1}{2}} D_{g} f^{\frac{n-1}{2}} .
$$

Note also the relationship between the two volume densities:

$$
d g_{p}=x^{n p} d g_{0}
$$

Together with the fact that the metric on $\Sigma$ is the same for all $p$, we see that the map

$$
\mathcal{C}_{c}^{\infty}\left(X, \Sigma, g_{0}\right) \rightarrow \mathcal{C}_{c}^{\infty}\left(X, \Sigma, g_{p}\right), \quad \phi \mapsto x^{-\frac{n p}{2}} \phi
$$

is an isometry with respect to the $L^{2}$ inner product. Let $A_{p}:=$ $x^{\frac{n p}{2}} D_{p} x^{-\frac{n p}{2}}$ be the conjugate of $D_{p}$ under this isometry. By using (6) with $f=x^{p}$, we get $A_{p}=x^{-\frac{p}{2}} D_{p} x^{-\frac{p}{2}}$. The commutation formula $\left[D_{0}, \mu\right]=c(d \mu)$ for $\mu \in \mathcal{C}^{\infty}(X)$ ends the proof.

In particular, the spectra of the closure of $D_{p}$ and $A_{p}$ are the same. It is therefore enough to study $A_{p}$, which is a cusp operator in the sense of the next section.

\section{REVIEW OF CUSP OPERATORS}

Definition 8. The algebra $\mathcal{D}_{c}(\bar{X})$ of cusp differential operators is the universal enveloping algebra of the Lie algebra ${ }^{c} \mathcal{V}(\bar{X})$ of cusp vector fields on $\bar{X}$.

The definition extends easily to cusp operators acting on sections of smooth vector bundles over $\bar{X}$.

The spectral properties of cusp differential operators acting as unbounded operators in $L^{2}\left(X, g_{0}\right)$ are well-understood, thanks to the calculus of cusp pseudo-differential operators of Melrose, a cousin of the celebrated $b$-algebra. We review below several results about cusp operators, and then use them to derive the spectral properties of a class of differential operators on $L^{2}\left(X, g_{p}\right)$ which includes the $p$-Dirac operator in the spin case. The cusp algebra is described e.g., in [24, Appendix A]. It is a particular case of the fibered cusp algebra [21] when the boundary fibers over a point, and of the cusp algebra on

manifolds with corners [15] when the corners are of codimension 1 . 
4.1. The principal symbol. Let $A$ be a cusp differential operator. Then the principal symbol of $A$, which lives a priori on the cotangent bundle to $\bar{X}$, lifts in fact to $T^{*} \bar{X}$ through the (dual) canonical map $T^{*} \bar{X} \rightarrow T^{*} \bar{X}$. We call this lifted symbol the cusp principal symbol, or simply the principal symbol when no confusion can occur.

4.2. The normal operator. This is a "boundary symbol" map, associating to any cusp operator $A \in \mathcal{D}_{c}(\bar{X}, \mathcal{E}, \mathcal{F})$ a family of differential operators on $M$ with one real polynomial parameter $\xi$ as follows:

$$
\mathcal{N}(A)(\xi)=\left.\left(e^{\frac{i \xi}{x} A e^{-\frac{i \xi}{x}}}\right)\right|_{M}
$$

where restriction to $M$ is justified by the mapping properties

$$
\begin{array}{r}
A: \mathcal{C}^{\infty}(\bar{X}, \mathcal{E}) \rightarrow \mathcal{C}^{\infty}(\bar{X}, \mathcal{F}) \\
A: x \mathcal{C}^{\infty}(\bar{X}, \mathcal{E}) \rightarrow x \mathcal{C}^{\infty}(\bar{X}, \mathcal{F})
\end{array}
$$

and by the isomorphism $\mathcal{C}^{\infty}(M)=\mathcal{C}^{\infty}(\bar{X}) / x \mathcal{C}^{\infty}(\bar{X})$. For example, $\mathcal{N}\left(i x^{2} \partial_{x}\right)(\xi)=-\xi$. The normal operators depends on $x$, nevertheless if we change $x$ inside its cusp structure, $x^{\prime}=a x+B x^{2}$ with $a>0$ constant and $B \in \mathcal{C}^{\infty}(\bar{X}, \mathbb{R})$, then the normal operator changes by a conjugation:

$$
\mathcal{N}^{\prime}(A)(\xi)=e^{-i b \xi / a^{2}} \mathcal{N}(A)(\xi / a) e^{i b \xi / a^{2}}
$$

where $b:=B_{\mid M}$. In particular, the invertibility of the normal operator for all values of $\xi \in \mathbb{R}$ is independent on the particular boundarydefining function $x$.

4.3. Cusp Sobolev spaces. For $k \in \mathbb{N}$, the cusp Sobolev space $H_{c}^{k}(\bar{X}, S)$ is defined as the space of those sections $\phi \in L^{2}(\bar{X}, S)$ such that $P(\phi) \in L^{2}(\bar{X}, S)$ for all $P \in \mathcal{D}_{c}^{k}(\bar{X}, S)$. It is a Hilbertable space in which $\mathcal{C}_{c}^{\infty}(\bar{X}, S)$ sits as a dense subspace; moreover, for all $a \in \mathbb{R}$ an operator $A \in \mathcal{D}_{c}^{k}(\bar{X}, \mathcal{E}, \mathcal{F})$ has the mapping property

$$
A: x^{a} H_{c}^{k}(\bar{X}, \mathcal{E}) \rightarrow x^{a} L_{c}^{2}(\bar{X}, \mathcal{F})
$$

which justifies the definition

$$
\mathcal{D}_{c}^{k, a}:=x^{-a} \mathcal{D}_{c}^{k}, \quad H_{c}^{k, a}:=x^{a} H^{k} .
$$

4.4. The cusp calculus. As part of his larger program of quantizing singular structures, Melrose constructed a calculus $\Psi_{c}^{z, s}(X)$ of pseudodifferential operators, $z, s \in \mathbb{C}$, in which $\mathcal{D}_{c}$ sits as the differential (i.e., local) operators. For the construction we refer the reader to [24]. Operators in $\Psi_{c}^{z, s}(X)$ extend by duality to distributions and map $H_{c}^{k, a}(X)$ 
into $H_{c}^{k-\Re(z), a-\Re(s)}(X)$. The cusp-principal symbol and the normal operator extend from $\mathcal{D}_{c}(X)$ to this larger calculus. The normal operator takes as values certain families of pseudodifferential operators on $M$, called suspended operators [23].

Definition 9. An operator $A \in \Psi_{c}^{z, s}(X)$ is called fully elliptic if the cusp principal symbol $\sigma_{z}\left(x^{s} A\right)$ is invertible outside the zero section in $T^{*} \bar{X}$, and if moreover $\mathcal{N}\left(x^{s} A\right)(\xi)$ is invertible as an operator from $H^{\Re(z)}(M)$ to $L^{2}(M)$ for all values of the parameter $\xi$.

Fully elliptic operators admit parametrices with respect to the two symbol structures. By a standard argument we get

Lemma 10. Let $A \in \Psi_{c}^{z, s}(X)$ be fully elliptic. Then the domain of the closure of $A$ inside $L_{c}^{2}(X)$ is $H_{c}^{\max \{\Re(z), 0\}, \max \{\Re(s), 0\}}(X)$. If moreover $A$ is symmetric then it is essentially self-adjoint.

The following lemma is the basic tool for the analysis of the spectrum of cusp operators.

Lemma 11 (Cusp Rellich lemma). Let $\bar{X}$ be a compact manifold with boundary, $x$ a boundary-defining function and $g_{0}$ a cusp metric on $X$. Then for $p, k \in \mathbb{R}$ the inclusion $x^{p} H_{c}^{k}(\bar{X}) \hookrightarrow L_{c}^{2}(\bar{X})$ is compact if and only if $p>0$ and $k>0$.

Proof. Easy, using a partition of unity and the classical Rellich lemma on a compact exhaustion of a neighborhood of infinity in $X$.

Corollary 12 (Melrose). Let $z, s \in \mathbb{C}, a, b \in \mathbb{R}$ and $A \in \Psi_{c}^{z, s}(\bar{X}, \mathcal{E}, \mathcal{F})$. Then $A$ is continuous as an operator

$$
A: H_{c}^{a+\Re(z), b+\Re(s)}(\bar{X}, \mathcal{E}) \rightarrow H_{c}^{a, b}(\bar{X}, \mathcal{F}),
$$

and it is Fredholm if and only if it is fully elliptic.

Corollary 13. The spectrum of a symmetric, fully elliptic operator $A \in \Psi_{c}^{z, s}(X, S)$ is discrete and accumulates towards infinity if and only if $\Re(z)>0$ and $\Re(s)>0$.

\section{Complex POWERS AND Eigenvalue Growth}

Analytic families of cusp operators have been introduced in [24] to define trace functionals in the spirit of Wodzicki's residue and to derive an index formula. This idea has been exploited in [15] for cusp manifolds with corners, and in [17] for fibered-cusp metrics. We need here a variant of Melrose' and Nistor's results. 
Proposition 14. Let $p \in \mathbb{R}_{+}^{*}$ and $\mathbb{C} \supset \Omega \ni z \mapsto A(z) \in \Psi_{c}^{z, p z}(X, S)$ an analytic family of cusp operators indexed by a connected open subset $\Omega \subset \mathbb{C}$. Then the trace map

$$
\{z \in \Omega ; \Re(z)<-n, \Re(p z)<-1\} \ni z \mapsto \operatorname{Tr}(A(z))
$$

is holomorphic, and extends meromorphically to $\Omega$ with (at most double) possible poles occurring at the superposition of the sets $(\mathbb{N}-n)$ and $(\mathbb{N}-1) / p$.

The first occurring pole is

- simple at $z=-n$, if $n>1 / p$, with residue

$$
-\frac{1}{(2 \pi)^{n}} \int_{{ }_{C S} \bar{X}^{*}} \operatorname{tr} \sigma_{-n} A(-n) ;
$$

- double at $z=-n$ if $n=1 / p$, with leading coefficient

$$
\frac{n}{(2 \pi)^{n}} \int_{{ }^{*} S^{*} \bar{X}_{\mid M}} \operatorname{tr} \sigma_{-n}\left(\mathcal{N}\left(x^{-1} A(-n)\right)\right) ;
$$

- simple at $z=-1 / p$, if $n<1 / p$, with residue

$$
-\frac{n}{2 \pi} \int_{\mathbb{R}} \operatorname{Tr} \mathcal{N}\left(x^{-1} A(-1 / p)\right)(\xi) d \xi .
$$

Proof. (sketch) By an analytic family of operators of varying order we mean that $A$ is holomorphic at $z \in \Omega$ inside the Banach space of bounded operators $B\left(H_{c}^{k, b}, L_{c}^{2}\right)$ for $k>\Re(z), b>p \Re(z)$.

For a trace-class cusp operator $A$ we write its trace as the integral of the Schwartz kernel of $A$ on the diagonal in the cusp double-space [24]. By the Fourier inversion formula, this becomes the integral on $T^{*} \bar{X}$ of the pointwise trace of the full symbol of $A$, times the (singular) canonical volume form given by the cusp symplectic form. Then we try to extend this integral meromorphically beyond the critical $z$. This extension follows by a standard elementary argument from the polyhomogeneity of the symbol at the boundary of the unit ball bundle, see [24] or [15]. Along the way we collect the information about the leading coefficient in the first pole (we could give all asymptotic coefficients in the same way, however only the first one matters in the rest of the paper). We have omitted the canonical volume forms on ${ }^{c} S^{*} \bar{X}$, resp. ${ }^{c} S^{*} \bar{X}_{\mid M}$, which are obtained from the symplectic volume form by contraction with the canonical radial, respectively cusp-normal vector field to $M$. 
Proposition 15. Let $A \in \Psi_{c}^{a, b}(X, \mathcal{E}), a, b>0$, be fully-elliptic, symmetric (hence self-adjoint) and positive. Then the complex powers $A^{z}$ form an analytic family as in Proposition 14.

Proof. (sketch) This is a particular case of [16, Thm. 7.1]. Here is the idea of the proof. Recall that for classical pseudodifferential operators on a closed manifold this was shown by Seeley [27]. Guillemin [11] gave another proof for scalar operators, which was extended by Bucicovschi to operators on vector bundles, or more generally with symbols taking values in a von Neumann algebra. This last method extends to algebras with "symbols" taking values in algebras like the suspended algebras, where the existence of complex powers is known. See also the recent paper [1] where complex powers are constructed in a more general framework.

Let $0<\lambda_{1} \leq \lambda_{2} \leq \ldots$ be the eigenvalues of $A$. The previous two propositions shows that the map

$$
z \mapsto \sum_{j=1}^{\infty} \lambda_{j}^{-z}=\operatorname{Tr}\left(A^{-z}\right)
$$

is holomorphic for large real parts of $z$, extends meromorphically to the complex plane with poles on the real axis, and describes the first pole. To deduce the asymptotic behaviour of $\lambda_{j}$ we use a result due to Delange [9, Theorem III]:

Lemma 16. Let $k \in \mathbb{N}^{*}, a \in \mathbb{R}_{+}^{*}$ and $0<\lambda_{1} \leq \lambda_{2} \leq \ldots$ be a nondecreasing sequence such that the series

$$
\sum_{j=0}^{\infty} \lambda_{j}^{-z}
$$

is absolutely convergent for $\Re(z)>a$. Let $\zeta(z)$ denote the sum of this series, thus $\zeta(z)$ is holomorphic for $\{\Re(z)>a\}$. Assume that $\zeta$ extends to a meromorphic function in a neighborhood of $\{\Re(z) \geq a\}$, regular except at $z=a$ where it has a pole of order $k$. Let $C$ be the coefficient of $(z-a)^{-k}$ in the Laurent expansion of $\zeta$ around $z=a$. Then the counting function $N(\lambda):=\max \left\{j \in \mathbb{N} ; \lambda_{j}<\lambda\right\}$ satisfies

$$
N(\lambda) \approx \frac{C}{a \cdot(k-1) !} \lambda^{a}(\log \lambda)^{k-1} .
$$

Proof. Set $\alpha(t):=\sum_{j=1}^{\infty} H\left(t-\log \lambda_{j}\right)$, where $H$ denotes the Heaviside function. Then

$$
f(z):=\int_{0}^{\infty} e^{-z t} \alpha(t) d t=\frac{1}{z} \zeta(z)
$$


satisfies the hypothesis of $[9$, Theorem III] with leading coefficient $C / a$ at $z=a$. Thus

$$
N\left(e^{t}\right)=\alpha(t) \approx \frac{C / a}{(k-1) !} e^{a t} t^{k-1}
$$

We note here that Karamata's method applied to the zeta function gives a weaker result than Theorem 3 , in terms of the asymptotics of the spectral function

$$
I(n)=\sum_{\lambda_{j}<n} \frac{1}{\lambda_{j}}
$$

This is because Karamata's proof does not take into account the behavior of the zeta function outside the real line.

We obtain as a corollary a general result about $p$-operators.

Theorem 17. Let $X$ be the interior of a compact manifold with boundary $\bar{X}$, with metric $g_{p}$ given by (4), $p \in \mathbb{R}$. Let $\Sigma$ be a hermitian vector bundle over $\bar{X}, q>0$ and $D \in \Psi_{c}^{1, q}(X, \Sigma)$ a symmetric fully elliptic cusp operator. Then $D$ is essentially self-adjoint on $\mathcal{C}_{c}^{\infty}(X, \Sigma)$ with discrete spectrum, and the counting function of its eigenvalues satisfies

$$
N(\lambda) \approx\left\{\begin{array}{cc}
C \lambda^{n} & \text { for } 1 / n<q \\
C \lambda^{n} \log \lambda & \text { for } q=1 / n \\
C \lambda^{1 / q} & \text { for } 0<q<1 / n .
\end{array}\right.
$$

The constant $C$ is explicitly given by Proposition 14 and Lemma 16.

Proof. $D_{q}$ is isometric to $A:=x^{n p / 2} D x^{-n p / 2} \in \Psi_{c}^{1, q}(X, \sigma)$ acting in $L^{2}\left(X, \sigma, g_{0}\right)$ as in Proposition 7. Now $\mathcal{N}\left(x^{q} A\right)=\mathcal{N}\left(x^{q} D\right)$ by the multiplicativity of the normal operator. So $A$ is also fully elliptic. The theorem follows from Corollary 13 and the results of this section.

Notably, the invariance of the normal operator under conjugation by powers of $x$ breaks down for $b$-operators. As a result, the analysis of conical singularities is more delicate.

\section{The Dirac operator of a CUSP Metric}

Let us return to the setting of Section 3. Assume that $\left(X, g_{0}\right)$ is spin, and fix a spin structure $P_{\text {Spin }}(X) \rightarrow P_{S O}(X)$. Using Lemma 5 we extend $P_{\text {Spin }}(X)$ to a spin structure for the Riemannian bundle $\left({ }^{C} T \bar{X}, g_{0}\right)$. We claim that the Dirac operator $D_{0}$ associated to the cusp metric $g_{0}$ given by (2) is a cusp differential operator (of order 1). For this we first need to describe the smooth structure of the spinor bundle over 
$\bar{X}$. The problem is that $g_{0}$ is singular over the boundary of $\bar{X}$. Choose a product decomposition

$$
\imath: M \times[0, \epsilon) \rightarrow \bar{X}
$$

near $M$ such that $x$ becomes the variable in $[0, \epsilon)$, i.e., $x(\imath(y, t))=t$. Then $h^{X}$ (see (5)) defines a Riemannian metric on the bundle $T M \times$ $[0, \epsilon)$, such that induced map $\imath: T M \times[0, \epsilon) \rightarrow{ }^{T} T \bar{X}$ is an isometric injection.

Choose a local orthonormal frame on $M \times\{0\}$, say $Y_{1}, \ldots, Y_{n-1}$. Let $Y_{1}(x), \ldots, Y_{n-1}(x)$ be the orthonormal frame obtained by the GrammSchmidt procedure from $Y_{1}, \ldots, Y_{n-1}$, viewed as a local frame on $M \times$ $\{x\}$. Let $Y_{0}$ be inward-pointing unit vector field normal to $M \times\{x\}$ for $0<x<\epsilon$. Again by (2), $Y_{0}$ extends to a smooth cusp vector field down to $x=0$. Thus we have found a local orthonormal frame in $\left({ }^{T} \bar{X}, g_{0}\right)$ near $x=0$ which defines a smooth structure on the orthonormal frame bundle of $\left({ }^{C} T \bar{X}, g_{0}\right)$, and hence on the spin bundle $P_{\text {Spin }}\left({ }^{\top} T \bar{X}\right)$.

An oriented orthonormal frame in $T M \times[0, \epsilon)$ gives rise to a unique oriented orthonormal frame in $T \bar{X}$ by adding $Y_{0}$ as the first component. Thus we get a $S O(n-1)$-map between the principal frame bundles. Define a spin structure on $M \times\{x\}, 0 \leq x<\epsilon$ as the pull-back of the spin bundle of $\bar{X}$ via this map. From the definition there exists a $\operatorname{Spin}(n-1)$-injection $P_{\text {Spin }}(M \times\{x\}) \rightarrow P_{\text {Spin }}(\bar{X})$.

Let $\Sigma_{n}$ be the spinor representation of $\operatorname{Spin}(n)$. If $n$ is odd, then $\Sigma_{n}=$ $\Sigma_{n-1}=\Sigma_{n-1}^{+} \oplus \Sigma_{n-1}^{-}$, while for $n$ even, $\Sigma_{n}=\Sigma_{n-1} \oplus \Sigma_{n-1}$. For $n$ odd, the Clifford action of the extra vector $V_{0}$ on $\Sigma_{n}$ is given by $c_{\Sigma_{n}}^{0}=\left[\begin{array}{cc}i & 0 \\ 0 & -i\end{array}\right]$, and at the same time, $c_{\Sigma_{n}}^{j}=c_{\Sigma_{n}}^{0} c_{\Sigma_{n-1}}^{j}$ for $j=1, \ldots, n-1$. For $n$ even, $V_{0}$ acts as $c_{\Sigma_{n}}^{0}=\left[\begin{array}{cc}0 & -1 \\ 1 & 0\end{array}\right]$, while $c_{\Sigma_{n}}^{j}=\left[\begin{array}{cc}0 & c_{\Sigma_{n-1}}^{j} \\ c_{\Sigma_{n-1}}^{j} & 0\end{array}\right]$.

Let $\Sigma M \times\{x\}, \Sigma \bar{X}$ be the spinor bundles over $M \times\{x\}$, respectively $\bar{X}$. It follows from the above discussion that $\Sigma \bar{X}_{\mid M \times\{x\}}$ can be identified with $\Sigma M \times\{x\}$ (for $n$ odd), respectively with $\mathbb{R}^{2} \otimes \Sigma M \times\{x\}$ (for $n$ even) as smooth bundles over $\bar{X}$. We wish to compute $D_{0}$, the Dirac operator of the metric $g_{0}$, in terms of the Dirac operators $D^{h_{x}^{X}}$ on $M \times\{x\}$. First, like for all Dirac operators, $\sigma_{2}\left(D_{0}^{2}\right)(\xi)=g_{0}(\xi, \xi)$. Lemma 5 implies that $D_{0}^{2}$ (and hence $D_{0}$ ) is cusp-elliptic. 
Lemma 18. The Levi-Civita covariant derivative $\nabla$ on $\left(X, g_{0}\right)$ extends to a differential operator with smooth coefficients

$$
\nabla: \mathcal{C}^{\infty}\left(\bar{X},{ }^{\top} \bar{X}\right) \rightarrow \mathcal{C}^{\infty}\left(\bar{X}, T^{*} \bar{X} \otimes{ }^{\top} T \bar{X}\right)
$$

ProOF. Use the Koszul formula for $\nabla$ applied to vectors $Y_{j}$. Since ${ }^{\top} T \bar{X}$ is stable by Lie bracket and $g_{0}$ is a true metric on $T \bar{X}$, the assertion follows immediately.

Let $\tilde{Y}$ be a local section in the principal spin bundle over $\bar{X}$ which lifts the local orthonormal frame $\left(Y_{0}, \ldots, Y_{n-1}\right)$ constructed previously. Let $\phi$ be a local section in $\Sigma \bar{X}$. There exists $s: \bar{X} \rightarrow \Sigma_{n}$ smooth so that $\phi=[\tilde{Y}, s]$, where the square bracket denotes the equivalence class modulo $\operatorname{Spin}(n)$. In other words, $\phi$ is represented by $s$ in the trivialization given by $\tilde{Y}$. Recall that in such a trivialization, the LeviCivita covariant derivative of the spinor $\phi$ takes the form (at least outside the boundary):

$$
\nabla_{Y} \phi=Y(s)+\frac{1}{2} \sum_{0 \leq i<j \leq n-1} g_{0}\left(\nabla_{Y} Y_{i}, Y_{j}\right) c^{i} c^{j} \phi
$$

From Lemma 18 it follows that $\nabla$ maps $\mathcal{C}^{\infty}(\bar{X}, \Sigma \bar{X})$ to $\mathcal{C}^{\infty}\left(\bar{X},{ }^{C} T^{*} \bar{X} \otimes\right.$ $\Sigma \bar{X})$. For $n$ odd,

$$
\begin{aligned}
D_{0} \phi= & c^{0}\left(D^{h} \phi+Y_{0}(s)\right) \\
& +\frac{1}{2} \sum_{1 \leq i<j \leq n-1}\left\langle\nabla_{Y_{0}} Y_{i}, Y_{j}\right\rangle c^{0} c^{i} c^{j} \phi \\
& +\frac{1}{2} \sum_{1 \leq i \leq n-1}\left\langle\nabla_{Y_{i}} Y_{0}, Y_{i}\right\rangle c^{0} \phi-\frac{1}{2} c^{0} c\left(\nabla_{Y_{0}} Y_{0}\right) \phi .
\end{aligned}
$$

For $n$ even,

$$
\begin{aligned}
D_{0}^{+} \phi= & D^{h}+Y_{0}(s)+\frac{1}{2} \sum_{1 \leq i<j \leq n-1}\left\langle\nabla_{Y_{0}} Y_{i}, Y_{j}\right\rangle c^{i} c^{j} \phi \\
& +\frac{1}{2} \sum_{1 \leq i \leq n-1}\left\langle\nabla_{Y_{i}} Y_{0}, Y_{i}\right\rangle \phi-\frac{1}{2} c\left(\nabla_{Y_{0}} Y_{0}\right) \phi .
\end{aligned}
$$

and so $D_{0} \in \mathcal{D}_{c}^{1}(\bar{X}, \Sigma \bar{X})$.

We make now the additional hypothesis that the metric $g_{0}$ satisfies (3), i.e., it is an exact cusp metric [24].

Lemma 19. If $g_{0}$ is an exact cusp metric then

- for $n$ odd, $\mathcal{N}\left(D_{0}\right)(\xi)=c^{0}\left(D^{h}+i \xi\right)$; 
- for $n$ even, $\mathcal{N}\left(D_{0}\right)(\xi)=\left[\begin{array}{cc}0 & D^{h}-i \xi \\ D^{h}+i \xi & 0\end{array}\right]$.

Thus $D_{0}$ is fully elliptic if and only if $D^{h}$ is invertible.

Proof. Recall that $\mathcal{I}$ is the ideal $x \cdot{ }^{c} \mathcal{V}(\bar{X})=\operatorname{ker} \mathcal{N}$ inside ${ }^{c} \mathcal{V}(\bar{X})$. From the hypothesis $(3), Y_{0}=x^{2} \partial_{x}+\mathcal{I}$. It follows that $\mathcal{N}\left(Y_{0}\right)=i \xi$ and also $\left[Y_{0}, Y_{j}\right] \in \mathcal{I}$ for all $j$. The Koszul formula shows that $\nabla_{Y_{0}} Y_{i}, \nabla_{Y_{i}} Y_{0}$ and $\nabla_{Y_{0}} Y_{0}$ all belong to $\mathcal{I}$. Since $\mathcal{N}(\mathcal{I})=0$, formula $(7)$ gives the desired formula. Then clearly $\mathcal{N}\left(D_{0}\right)(\xi)$ is invertible for all $\xi \in \mathbb{R}$ if and only if $D^{h}$ is invertible.

For example, if $\operatorname{scal}_{h}(M) \geq 0$ and does not vanish identically on any connected component of $M$ then, by Lichnerowicz's formula, $D_{0}$ is fully elliptic.

\section{Proofs of the MAIN RESUlts}

In this section (3) is assumed to hold.

Proof of Theorem 1. By Proposition 7, we see that for each $p, D_{p}$ is isometric to an operator $A_{p} \in \mathcal{D}_{c}^{1, p}(\bar{X}, \Sigma)$ with the property that $A_{p}-x^{-p} D_{0} \in \mathcal{D}_{c}^{0, p-1}(\bar{X}, \Sigma)$. This means that $A_{p}$ is fully elliptic if and only $D_{0}$ is. Lemmata 10 and 19 end the proof.

Proof of Theorem 2. Assume that $D^{h}$ is invertible, so $D_{p} \in$ $\Psi_{c}^{1, p}(\bar{X}, S(\bar{X}))$ is fully elliptic by Lemma 19 . The result follows from Corollary 13.

Proof of Theorem 3. We assume that $p>0$ and $D^{h}$ is invertible. Then $A_{p}$ is fully elliptic and has discrete spectrum, and moreover $P_{\text {ker } A_{p}}$, the orthogonal projection onto the kernel of $A_{p}$ in $L^{2}\left(X, \Sigma, g_{0}\right)$, belongs to $\Psi_{c}^{-\infty,-\infty}(X, \Sigma)$. We apply Proposition 14 to the analytic family $A(z):=\left(A_{p}^{2}+P_{\text {ker } A_{p}}\right)^{z / 2} \in \Psi_{c}^{z, p z}(\bar{X}, \Sigma)$ to find that the zeta function of $A_{p}^{2}$ has either a simple or a double leading pole. Note that $\sigma_{1}(A(1))=\|\cdot\|_{g_{p}}$. From Lemma 19 we get

$$
\mathcal{N}\left(x^{-1} A(1)^{-1 / p}\right)(\xi)=\left(\left(D^{h}\right)^{2}+\xi^{2}\right)^{-\frac{1}{2 p}},
$$

for $n$ odd, respectively

$$
\mathcal{N}\left(x^{-1} A(1)^{-1 / p}\right)(\xi)=\left[\begin{array}{cc}
\left(\left(D^{h}\right)^{2}+\xi^{2}\right)^{-\frac{1}{2 p}} & 0 \\
0 & \left(\left(D^{h}\right)^{2}+\xi^{2}\right)^{-\frac{1}{2 p}}
\end{array}\right]
$$


for $n$ even. Thus

$$
\begin{aligned}
\operatorname{tr} \sigma_{-n} A(-n)_{\mid c S^{*} \bar{X}} & =\operatorname{dim} \Sigma(n)=2^{[n / 2]} \\
\operatorname{tr} \sigma_{-n}\left(\mathcal{N}\left(x^{-1} A(-n)\right)\right)_{\mid c S^{*} \bar{X}_{\mid M}} & =\operatorname{dim} \Sigma(n)=2^{[n / 2]} \\
\operatorname{tr} \mathcal{N}\left(x^{-1} A(-1 / p)\right)(\xi) & =\epsilon_{n} \operatorname{tr}\left(\left(D^{h}\right)^{2}+\xi^{2}\right)^{-\frac{1}{2 p}} .
\end{aligned}
$$

The last identity implies that

$$
\begin{aligned}
\int_{\mathbb{R}} \operatorname{Tr} \mathcal{N}\left(x^{-1} A(-1 / p)\right)(\xi) d \xi & =\epsilon_{n} \operatorname{Tr}\left(\left(D^{h}\right)^{2}\right)^{-\frac{1}{2 p}+\frac{1}{2}} \int_{\mathbb{R}}\left(1+\xi^{2}\right)^{-\frac{1}{2 p}} d \xi \\
& =\epsilon_{n} \zeta\left(D^{h}, \frac{1}{p}-1\right) \frac{\sqrt{\pi} \Gamma\left(\frac{1}{2 p}-\frac{1}{2}\right)}{\Gamma\left(\frac{1}{2 p}\right)}
\end{aligned}
$$

where the integral was evaluated using [26, equation (4)]. The result follows from these formulae, Proposition 14 and Lemma 16.

Theorem 3 applies with minimal modifications to Dirac operators twisted by a bundle $W \rightarrow \bar{X}$ with smooth connection down to $x=0$, provided that the induced twisted Dirac operator $D^{M, W}$ over $M$ is invertible. Namely, for $p \geq 1 / n$ we have to multiply by $\operatorname{dim}(W)$ the coefficient of $\lambda^{n}$, resp. $\lambda^{n} \log \lambda$, while for $0<p<1 / n$ we must replace $D^{h}$ by $D^{M, W}$ inside the zeta function. If $M$ is connected then the obstruction to the invertibility of $D^{M, W}$ given by index theory vanishes, as a consequence of cobordism invariance. Thus, by Anghel's result [2], $D^{M, W}$ is invertible for generic connections on $W$, at least if $\operatorname{dim}(M) \leq 4$.

\section{WEYL LAWS FOR GENERAL CUSP METRICS}

Let us consider the case of a general cusp metric. Write $g_{0}$ in the form (5) and fix a product decomposition near $M$ so that $\theta^{X}$ and $h^{X}$ are uniquely defined. Let $\theta^{\#}$ be the dual vector field to $\theta^{X}$ relative to $h^{X}$. Set

$$
F:=1 /\left\|x^{2} \partial_{x}-\theta^{\#}\right\|_{g_{0}}, \quad f:=F_{\mid M} \in \mathcal{C}^{\infty}(M) .
$$

Proposition 20. The normal operator of the Dirac operator corresponding to $g_{0}$ is given by

$$
\begin{aligned}
\mathcal{N}\left(D_{0}\right)(\xi)= & c^{0}\left(D^{h}+i f \xi-f \nabla_{\theta^{\#}}\right. \\
& \left.+\frac{1}{4} f c(d \theta)-\frac{1}{4} f \operatorname{tr}\left(L_{\theta \#} h(0)\right)-\frac{1}{2} c(d f) / f\right)
\end{aligned}
$$

for $n$ odd, respectively

$$
\begin{aligned}
\mathcal{N}\left(D_{0}^{+}\right)(\xi)= & D^{h}+i f \xi-f \nabla_{\theta^{\#}} \\
& +\frac{1}{4} f c(d \theta)-\frac{1}{4} f \operatorname{tr}\left(L_{\theta} h(0)\right)-\frac{1}{2} c(d f) / f
\end{aligned}
$$


for $n$ even.

Proof. The idea is to expand formula (7) for $D_{0}$. Note that $Y_{0}=$ $F\left(x^{2} \partial_{x}-\theta^{\#}\right)$, and therefore

$$
\begin{aligned}
{\left[Y_{i}, Y_{0}\right] } & =\frac{Y_{i}(F)}{F} Y_{0}+F\left(-\left[Y_{i}, \theta^{\#}\right]-x^{2} \partial_{x}\left(Y_{i}\right)\right) \\
& \equiv \frac{Y_{i}(f)}{f} Y_{0}-f\left[Y_{i}, \theta^{\#}\right]+\mathcal{I}^{2}
\end{aligned}
$$

(recall that $\mathcal{I}$ is the ideal $x^{c} \mathcal{V}(\bar{X})$ annulated by $\mathcal{N}$ ). Thus

$$
\begin{aligned}
\mathcal{N}\left(D_{0}\right)(\xi)= & c^{0} \cdot\left(D^{h}+i f \xi-f \nabla_{\theta}\right) \\
& +\frac{1}{2} \sum_{1 \leq i<j \leq n-1}\left\langle\nabla_{f x^{2} \partial_{x}} Y_{i}, Y_{j}\right\rangle_{\mid x=0} c^{0} c^{i} c^{j} \\
& +\frac{1}{2} \sum_{1 \leq i \leq n-1}\left\langle\nabla_{Y_{i}} Y_{0}, Y_{i}\right\rangle_{\mid x=0} c^{0} \\
& -\frac{1}{2} \sum_{j=1}^{n-1}\left\langle\nabla_{Y_{0}} Y_{0}, Y_{j}\right\rangle_{\mid x=0} c^{0} c^{j} .
\end{aligned}
$$

We already see here the first three terms from (8). Koszul's formula gives

$$
2\left\langle\nabla_{x^{2} \partial_{x}} Y_{i}, Y_{j}\right\rangle=Y_{i}\left(\theta_{j}\right)-Y_{j}\left(\theta_{i}\right)-\theta\left(\left[Y_{i}, Y_{j}\right]\right)=d \theta\left(Y_{i}, Y_{j}\right)
$$

so by summing over $i<j$ we get the fourth term. Since $Y_{i}$ has constant length 1 , it follows by (9) that

$$
\left\langle\nabla_{Y_{i}} Y_{0}, Y_{i}\right\rangle=\left\langle\left[Y_{i}, Y_{0}\right], Y_{i}\right\rangle=-f\left\langle\left[Y_{i}, \theta^{\#}\right], Y_{i}\right\rangle+\mathcal{I}^{2}
$$

Use

$$
\sum_{i=1}^{n-1}\left\langle\left[Y_{i}, \theta^{\#}\right], Y_{i}\right\rangle=\frac{1}{2} \operatorname{tr}\left(L_{\theta \#} h(0)\right)=\frac{L_{\theta \#} d h(0)}{d h(0)}
$$

to get the fifth term. As for the last term, again by (9) we have

$$
\left\langle\nabla_{Y_{0}} Y_{0}, Y_{j}\right\rangle=-\left\langle Y_{0}, \nabla_{Y_{0}} Y_{j}\right\rangle=\left\langle Y_{0},\left[Y_{j}, Y_{0}\right]\right\rangle \equiv Y_{j}(f) / f+\mathcal{I}^{2} .
$$

This settles the case $n$ odd. The case $n$ even is done in the same way.

We wish now to give conditions on $g_{0}$ so that $\mathcal{N}\left(D_{0}\right)(\xi)$ is invertible for all $\xi \in \mathbb{R}$. If $\theta=0$ this condition was seen to be equivalent to $D^{M, h(0)}$ 
being invertible. For the general case, we rewrite (8) as

$$
\begin{aligned}
& \mathcal{N}\left(D_{0}\right)(\xi)= \\
& \quad=c^{0} f\left(f^{-\frac{1}{2}} D^{h} f^{-\frac{1}{2}}+i \xi-\left(\nabla_{\theta \#}+\frac{1}{2} \frac{L_{\theta \#} d h(0)}{d h(0)}\right)+\frac{1}{4} c(d \theta)\right) .
\end{aligned}
$$

It is not clear if a reasonable condition on $h, f$ and $\theta$ exists so that the above operator is invertible for all $\xi \in \mathbb{R}$. Recall that the metric $h$ is not canonically determined by $g_{0}$, since it depends on the choice of $x$ inside the fixed cusp structure. Thus, we rewrite $\mathcal{N}\left(D_{0}\right)$ in terms of the canonical metric $g^{M}$ on $M$, see Lemma 6 , although we must still fix $x$ in order to define $\mathcal{N}$.

Proposition 21. Let $g_{0}$ be a cusp metric on $X$ and $\alpha, g^{M}, q$ defined in Lemma 6. Then for $n$ odd,

$$
\mathcal{N}\left(D_{0}\right)(\xi)=c^{0} q^{-\frac{n+1}{2}}\left(D^{g^{M}}+i \xi(1-c(\alpha))+\frac{1}{4} c(d \alpha)\right) q^{-\frac{n-1}{2}}
$$

while for $n$ even,

$$
\mathcal{N}\left(D_{0}^{+}\right)(\xi)=q^{-\frac{n+1}{2}}\left(D^{g^{M}}+i \xi(1-c(\alpha))+\frac{1}{4} c(d \alpha)\right) q^{-\frac{n-1}{2}} .
$$

PROOF. Recall that in the expression (5) for $g_{0}$, the tensors $\theta^{X}, h^{X}$ are well-defined only up to $\mathcal{I}^{2}$. Fix a product decomposition of $\bar{X}$ near $M$, thus removing the ambiguity.

First, assume that $a_{00}=1$ near $M$, so $g_{0}=\left(d x / x^{2}+\theta^{X}\right)^{2}+h^{X}-\theta^{X} \otimes \theta^{X}$. Let $\tilde{Y}_{0}:=x^{2} \partial_{x}$. Define an isometric embedding

$$
\left(T M \times[0, \infty), h^{X}\right) \rightarrow\left({ }^{\top} T \bar{X}, g_{0}\right), \quad Y \mapsto \tilde{Y}:=Y-\theta(Y) \tilde{Y}_{0}
$$

This allows us to compare spinors on $X$ and on $M$. Choose a local orthonormal frame $Y_{1}, \ldots, Y_{n-1}$ on $M$, transport it to $X$ using the product decomposition and re-orthonormalize it using GrammSchmidt. Note that the frame $\left\{Y_{j}\right\}$ is different from the frame with the same name from Section 6 , since they are orthonormal with respect to different metrics. Then $\tilde{Y}_{0}, \ldots, \tilde{Y}_{n-1}$ is a (smooth) orthonormal frame on $T \bar{X}$. We use $i, j, k$ to denote a subscript in $\{1, \ldots, n-1\}$. Notice that

$$
\begin{aligned}
{\left[\tilde{Y}_{i}, \tilde{Y}_{j}\right] } & \left.=\widetilde{\left[Y_{i}, Y_{j}\right.}\right]-d \theta\left(Y_{i}, Y_{j}\right)+\mathcal{I}^{2} \\
{\left[\tilde{Y}_{0}, \tilde{Y}_{i}\right] } & \in \mathcal{I}^{2} .
\end{aligned}
$$


So

$$
\begin{aligned}
\left(\nabla_{\tilde{Y}_{i}} \tilde{Y}_{j}, \tilde{Y}_{k}\right) & =\left(\nabla_{Y_{i}} Y_{j}, Y_{k}\right) \\
\nabla_{\tilde{Y}_{0}} \tilde{Y}_{0} & \in \mathcal{I}^{2} \\
\left(\nabla_{\tilde{Y}_{i}} \tilde{Y}_{0}, \tilde{Y}_{i}\right) & \in \mathcal{I}^{2} \\
\left(\nabla_{\tilde{Y}_{0}} \tilde{Y}_{j}, \tilde{Y}_{k}\right) & =\frac{1}{2} d \theta\left(\tilde{Y}_{i}, \tilde{Y}_{j}\right)+\mathcal{I}^{2} .
\end{aligned}
$$

Using (7), under the assumption that $a_{00}=1$ we get immediately in the case $n$ is odd

$$
\left.\mathcal{N}\left(D_{0}\right)(\xi)=c^{0}\left(D^{g^{M}}+i \xi(1-c(\theta))+\frac{1}{4} c(d \theta)\right)\right) .
$$

Remove now the assumption that $a_{00}=1$ in a neighborhood of $M$. Using the formula for the conformal change for Dirac operators (6), we get the desired expression. The case $n$ even is entirely similar.

As a corollary, we give a condition for the full ellipticity of $D_{p}$ in some cases where $g_{0}$ is not exact.

Corollary 22. Let $g_{0}$ be a cusp metric with $\alpha$ closed (by Lemma 6, this condition is independent on the boundary-defining function). Then $D_{p}$ is fully elliptic if and only if $D^{g^{M}}$ is invertible.

Proof. By Proposition 21, we must check the invertibility of the family of operators

$$
P(\xi):=D^{g^{M}}-i \xi c(\alpha)+i \xi
$$

for all $\xi \in \mathbb{R}$. But $P(\xi)$ is invertible if and only if $P^{*}(\xi) P(\xi)=\left(D^{g^{M}}-\right.$ $i \xi c(\alpha))^{2}+\xi^{2}$ is invertible. This holds automatically for $\xi \neq 0$, and for $\xi=0$ it is equivalent to $D^{g^{M}}$ being invertible.

Product cylindrical metrics have been studied by several authors starting with [3]. Such metrics can be considered either as $b$ - or as cusp metrics; they can actually be treated by elementary methods using separation of variables. Melrose [22] has successfully studied exact $b$-metrics, which are in some sense only asymptotically cylindrical. Corollary 22 allows even more geometric structure to be embedded in the metric. If $H^{1}(M, \mathbb{R}) \neq 0$, it simply says that our results on Weyl laws hold for some metrics (i.e., closed cusp metrics) which are not exact.

Let us finally state our most general result about Dirac eigenvalues of cusp metrics.

Theorem 23. Assume that the normal operator of $D_{0}$, computed in two different ways in Propositions 20, 21, is invertible. Then for $p>0$, 
$D_{p}$ is essentially self-adjoint with pure point spectrum accumulating towards infinity. The rate of growth is given by Theorem 3 if $p \geq 1 / n$, and by Theorem 17 if $0<p<1 / n$.

This theorem has the drawback that the invertibility of $\mathcal{N}\left(D_{0}\right)$ must be assumed. The only case where we have found a reasonable condition for invertibility is for closed cusp metrics (Corollary 22). We are therefore let to the following

Problem. Let $\left(M, g^{M}\right)$ be a connected compact Riemannian spin manifold and $\theta$ a 1 -form on $M$. Find conditions on $\theta, g^{M}$ so that the family of operators

$$
\left.D^{g^{M}}+i \xi(1-c(\theta))+\frac{1}{4} c(d \theta)\right)
$$

is invertible for all $\xi \in \mathbb{R}$.

Another variant of the problem would be to assume additionally that $M$ vanishes in the Spin bordism ring. Mere invertibility of $D^{g^{M}}$ is not enough in general, as shown by the following example (courtesy of Andrei Moroianu, see also [6]):

Example 24. Let $M=S^{2}$ with an arbitrary metric $h$ and $D$ the associated Dirac operator. Then $D^{h}$ is invertible, since the Dirac operator of the standard metric is invertible, and any two metrics on $S^{2}$ are conformally isometric. Nevertheless, there exist a metric $h$ and $\theta \in \Lambda^{1}\left(S^{2}\right)$ so that $D^{h}+c(\theta)$ is not invertible. For this, take $h$ to be the metric induced from an immersion of $S^{2}$ in $\mathbb{R}^{3}$ with total mean curvature 0 . First, there exists such an immersion, since we can deform $S^{2}$ by stretching cylinders of positive or negative mean curvature, thus increasing or decreasing the total mean curvature at will. Secondly, take $\tilde{\phi}$ to be a constant spinor on $\mathbb{R}^{3}$, and pull it back to a spinor $\phi=\left(\phi^{+}, \phi^{-}\right)$on $M$. Then by [10, Proposition 2$]$,

$$
D^{h} \phi^{+}=-i H \phi, \quad D^{h} \phi^{-}=i \phi^{+}
$$

where $H$ is the mean curvature function of $(M, h)$. The form $H d h$ has volume 0 , thus it is exact, i.e., $H d h=d \theta$ for some $\theta \in \Lambda^{1}(M, \mathbb{R})$. Recall moreover that $c(d h)$ acts by $\pm i$ on $\Sigma^{ \pm}(M)$. Thus $\phi$ is a solution of the equation

$$
\left(D^{h}+c(d \theta)\right) \phi=0
$$




\section{REFERENCES}

[1] B. Ammann, R. Lauter, V. Nistor and A. Vasy, Complex powers and noncompact manifolds, math.OA/0211305

[2] N. Anghel, Generic vanishing for harmonic spinors of twisted Dirac operators, Proc. Amer. Math. Soc. 124 (1996), 3555-3561.

[3] M.F. Atiyah, V.K. Patodi and I.M. Singer, Spectral asymmetry and Riemannian geometry. I, Math. Proc. Cambridge Philos. Soc. 77 (1975), 43-69.

[4] C. Bär, The Dirac operator on hyperbolic manifolds of finite volume, J. Differential Geom. 54 (2000), 439-488.

[5] C. Bär and M. Dahl, Surgery and the spectrum of the Dirac operator, J. Reine Angew. Math. 552 (2002), 53-76.

[6] C. Bär, P. Gauduchon and A. Moroianu, Generalized Cylinders in SemiRiemannian and Spin Geometry, math.DG/0303095.

[7] B. Bucicovschi, An extension of the work of V. Guillemin on complex powers and zeta functions of elliptic pseudodifferential operators, Proc. Amer. Math. Soc. 127, (1999), 3081-3090.

[8] T. Christiansen and M. Zworski, Spectral asymptotics for manifolds with cylindrical ends, Ann. Inst. Fourier (Grenoble) 45 (1995), 251-263.

[9] H. Delange, Généralization du théorème de Ikehara, Ann. Sci. Ecole Norm. Sup. (3) 71 (1954), 213-242.

[10] T. Friedrich, On the spinor representation of surfaces in Euclidean 3-space, J. Geom. Phys. 28 (1998), 143-157.

[11] V. Guillemin, A new proof of Weyl's formula on the asymptotic distribution of eigenvalues, Adv. in Math. 55 (1985), 131-160.

[12] N. Hitchin, Harmonic spinors, Adv. in Math. 14 (1974), 1-55.

[13] L. Hörmander, The spectral function of an elliptic operator, Acta Math. 121 (1968), 193-218.

[14] S. Ikehara, An extension of Landau's theorem in the analytic theory of numbers, Journ. Math. Phys. MIT 10 (1931), 1-12.

[15] R. Lauter and S. Moroianu, The index of cusp operators on manifolds with corners, Ann. Global Anal. Geom. 21 (2002), 31-49.

[16] R. Lauter and S. Moroianu, Homology of pseudodifferential operators on manifolds with fibered cusps, T. Am. Math. Soc. 355 (2003), 3009-3046.

[17] R. Lauter and S. Moroianu, An index formula on manifolds with fibered cusp ends, math.DG/0212239.

[18] M. Lesch and N. Peyerimhoff, On index formulas for manifolds with metric horns, Comm. Partial Differential Equations 23 (1998), 649-684.

[19] P. Loya, Tempered operators and the heat kernel and complex powers of elliptic pseudodifferential operators, Comm. Partial Differential Equations 26 (2001), 1253-1321.

[20] S. Maier, Generic metrics and connections on Spin- and Spin ${ }^{c}$-manifolds, Comm. Math. Phys. 188 (1997), 407-437.

[21] R. R. Mazzeo and R. B. Melrose, Pseudodifferential operators on manifolds with fibered boundaries, Asian J. Math. 2 (1998), 833-866.

[22] R. B. Melrose, The Atiyah-Patodi-Singer index theorem, Research Notes in Mathematics 4, A. K. Peters, Wellesley, MA, 1993.

[23] R. B. Melrose, The eta invariant and families of pseudodifferential operators, Math. Res. Letters 2 (1995), 541-561. 
[24] R. B. Melrose and V. Nistor, Homology of pseudodifferential operators I. Manifolds with boundary, funct-an/9606005.

[25] S. Minakshisundaram, A. Pleijel, Some properties of the eigenfunctions of the Laplace operator on Riemannian manifolds, Canad. J. Math. 1 (1949), 242-256.

[26] S. Moroianu, Adiabatic limits of eta and zeta functions of elliptic operators, to appear in Math. Z., math.DG/0204163.

[27] R. Seeley, Complex powers of an elliptic operator, Proc. Sympos. Pure Math. (Singular integrals), Chicago, Ill., 1966, Amer. Math. Soc. (1967), 288-307.

Institutul de Matematică al Academiei Române, P.O. Box 1-764, RO70700 Bucharest, Romania

Université Paul Sabatier, UFR Mig, 118 route de Narbonne, 31062 Toulouse, France

E-mail address: moroianu@alum.mit.edu 